\title{
Correspondence
}

\section{Training course in behavioural psychotherapy DeAR SIR,}

It is not altogether clear whether Dr Taylor's letter in your August issue (p 122-3) can be referring to the course advertized in your March Bulletin. He mentions a 'proposed Institute of Psychiatry course' while that advertized is organized by the AUTP in conjunction with the Institute of Psychiatry. The letter implies that the course restricts itself to anxietyrelated disorders and treatment by exposure and response prevention, and that it omits operant learning and behaviour disorders in adults and children. Our course in fact includes the behavioural treatment of sexual, marital and family problems, children's problems, enuresis, obesity, stuttering, mental handicap, social skills training and inappropriate parenting.

Dr Taylor's letter stresses the importance of behavioural treatment of alcoholism and drug addiction, but this is of unproven value and would be out of place in an introductory course. The AUTP course is designed to teach a healthy respect for clinical realities and a wariness of inflated claims for behavioural methods as a panacea for all ills, whereas we view the approach as only useful for appropriately selected problems. It would also be interesting to see Dr. Taylor's evidence for the necessity for wide theoretical knowledge to produce capable behavioural clinicians in the face of much data to the contrary. He asserts that the subject is best taught by practioners outside psychiatry. Our course in fact includes training not only by psychiatrists but also by three clinical psychologists and a nurse-therapist, and reflects the interdisciplinary nature of the AUTP. A team approach seems preferable to a narrow arrogation to one profession of all purported expertise in this field; especially given $\mathrm{Dr}$ Taylor's contention that 'the theories and methods, the entire conceptual basis of behaviour therapy are at variance with those of the rest of psychiatry'. We take the view that behavioural psychotherapy is but one aspect of good clinical management, and teach it as such. Dr Taylor's 'broadspectrum behavioural treatment programme based on a full functional analysis of the patient in his environment' is in our view part of this approach.

As for the 'serious dilution in the standards of training offered within the NHS', we would be interested to know where such training exists, given its concealment from the AUTP. Partisan and parochial claims by particular professions and regions could detract from the healthy growth of the behavioural field. It is our hope that a sober interdisciplinary introduction such as we offer will help experienced clinicians concerned with postgraduate training to develop expertise which will in time enable them to become trainers aware of the limitations as well as the strengths of the behavioural approach.
We would welcome greatly the development of a further, improved course by Dr Taylor, perhaps in Glasgow, and would be happy to collaborate in any way possible.

JoHn COBB

Michael Crowe

ISAAC MARKS

Institute of Psychiatry

De Crespigny Park

London SES 6AF

\section{Medical standards of finness to ditre heavy goods velicles}

DeAR Sir,

At the invitation of the Department of Transport, a small working party has considered the medical standards which should be applied to drivers of heavy goods vehicles.

There is no provision at the present time for continuously monitoring an HGV driver's health. Train drivers and airline pilots are subject to medical assessment after a period of illness, and London Transport arranges medical examinations for bus drivers after any period of sick absence exceeding 21 days. Heavy goods vehicles are defined as the largest commercial vehicles carrying loads in excess of 7.5 metric tonnes laden weight. There are 850,000 HGV licence holders, 80,000 of whom are self-employed. The number of registrations of these vehicles is on the increase. The vehicles carry all varieties of cargoes: from lethal ones such as nerve gas (organic phosphorus compounds used in agriculture) to others as benign as a load of hay. It has been suggested that some of the mixed chemical cargoes carried in drums are capable of so interacting as to destroy an entire community. There is apparently no way of restricting a heavy goods vehicle driver's licence to non-dangerous cargoes. The Department of Transport has had discussions with the Health \& Safety Executive and also with the Home Office, and both agree that a discrimination against dangerous substances would be impossible to enforce. The drivers of these vehicles may spend long hours at the wheel and are oftell subject to such special stresses as maintaining delivery schedules, working against the clock and combating varying traffic and weather conditions. The current psychiatric standards applied to HGV drivers are those recommended by Professor T. C. N. Gibbens on page 33 of the 1976 edition of 'Medical Aspects of Fitness to Drive', and we found them a very useful basis for our discussion. Persons with a history of psychosis or who need continuing medication with psychotropic drugs are not recommended for HGV driving licences. The application of Professor Gibbens' advice has not led to practical difficulties, although each case has to be very carefully considered individually. 